\section{Wavelength stability optimization of L-band superfluorescent fiber source}

\author{
Xiulin Wang \\ Jimei University \\ Department of Physics \\ Xiamen, 361021, China

\section{Wencai Huang} \\ Xiamen University \\ Department of Electronic Engineering \\ Xiamen, 361005, China
}

\begin{abstract}
A new bidirectional pumped configuration for an L-band erbium-doped superfluorescent fiber source is demonstrated. Data are presented showing that with this configuration, the mean wavelength variation with the pump power can be reduced to zero by optimizing the fiber length and the pumping ratio of forward to total pump power. (c) 2005 Society of Photo-Optical Instrumentation Engineers. [DOI: 10.1117/1.1928271]
\end{abstract}

Subject terms: fluorescence; fiber optics; erbium lasers; fiber optic sensors.

Paper L040860RR received Nov. 30, 2004; revised manuscript received Jan. 19, 2005, and Apr. 12, 2005; accepted for publication Apr. 19, 2005; appeared online Apr. 19, 2005; published online Jun. 16, 2005.

\section{Introduction}

Superfluorescent fiber source (SFS) using amplified spontaneous emission (ASE) from an erbium-doped fiber (EDF) has been a topic of continuing research because of its wide range of applications. ${ }^{1,2}$ In particular, in fiber optic gyroscope (FOG) applications, the mean wavelength stability of the SFS is vital since it determines the accuracy of rotation detection of the FOG. On the other hand, the bandwidth is another important parameter since a SFS with a broad bandwidth implies that a higher SNR would be obtained from the FOG. In the past decades, various designs of SFSs have been demonstrated with the aim to improve these two performance parameters. ${ }^{3-5}$ However, all of the highstability SFSs reported so far were restricted to the C-band SFS. Although it has the potential for a flatter spectrum, there are no reports on a high-stability L-band SFS because both theoretical and experimental results show that it cannot have pump-power-independent mean-wavelength operation with a single laser pump. ${ }^{1,6}$ In this letter, we demonstrate a new configuration for the mean-wavelength stability L-band SFS. A single laser diode has replaced the pair of pump diodes used in Ref. 7 to avoid the pump power variation of backward pump or forward pump, respectively. As a result, the mean-wavelength variation with pump power can be reduced to zero by optimizing the fiber length and the pumping ratio of forward to total pump power.

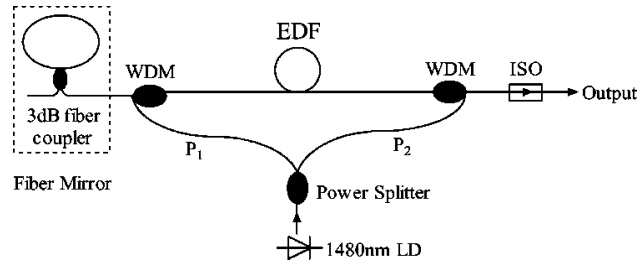

Fig. 1 Proposed configuration for mean-wavelength stability L-band SFS.

$\mathbf{L}$

E

\section{Configuration and Results}

The suggested configuration of the L-band erbium-doped SFS is shown in Fig. 1, which consists of a section of EDF, two 1480/1590-nm wavelength division multiplexers (WDMs), a 1480-nm pump laser diode (LD), a power splitter used to divide the pump power into two portions, a fiber mirror with nearly $100 \%$ reflectivity made by a $3-\mathrm{dB}$ fiber couple, and an optical isolator (ISO) at the output port. The fiber mirror is used to reflect the ASE, then causes the wide and flat L-band ASE light to emerge from the output port. Therefore, this SFS design is a double-pass bidirectional pumped configuration. The EDF used in both the numerical simulations and experiments was a Lucent Technologies heavily doped LRL fiber with a peak absorption of 27-33 $\mathrm{dB} / \mathrm{m}$ at $1530 \mathrm{~nm}$.

As the spectral wavelength range and flatness of the SFS with bidirectional pump are length dependent, to obtain the L-band output, the EDF length should be selected properly in order to make the ASE generated by the forward pump move to L-band. An optimum length of $19 \mathrm{~m}$ of LRL fiber is suitable for the double-pass bidirectional pumped L-band SFS configuration to obtain the flattest L-band SFS spectrum by the previous simulation. ${ }^{8}$

Figure 2 illustrates the calculated mean-wavelength variation and the pump efficiency versus pumping ratio $\left(R_{p}=P_{\text {forward }} / P_{\text {total }}\right)$ for the total pump power fixed at 100 $\mathrm{mW}$. Commercial EDF optical amplifier simulation software was used for the simulations. ${ }^{9}$ The rate equations, power evolution equations, and boundary conditions of the SFS configuration were completed by the simulation software. The software was used to characterize SFS and is shown to be effective. ${ }^{5}$ It is found from the results that two pumping ratios exist where the mean wavelength is independent of the small variations in the power splitting ratio of the power splitter. These two pumping ratios correspond to $R_{p}=0.2$ and 0.5 . These two values are significant because the splitting ratio may have small variations with the

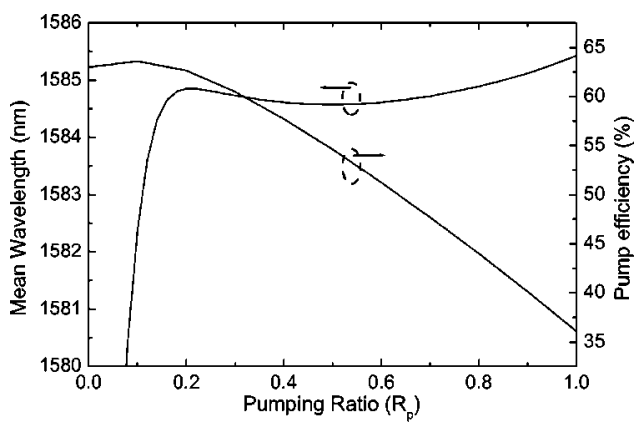

Fig. 2 Calculated mean wavelength and pump efficiency versus pumping ratio with the total pump power fixed at $100 \mathrm{~mW}$. 


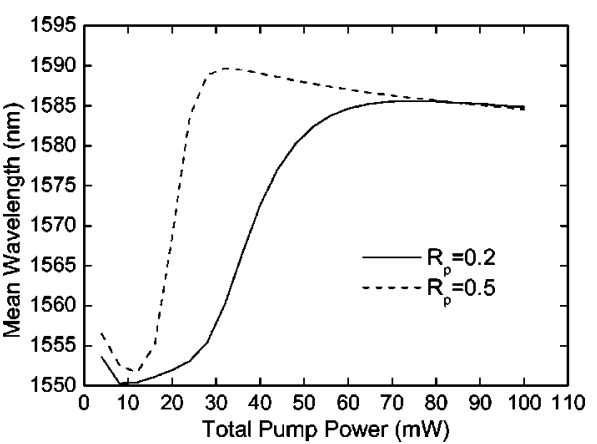

Fig. 3 Calculated mean wavelength as a function of the total pump power with $R_{p}=0.2$ and 0.5 .

ambient temperature and total pump power, etc. Figure 2 also shows that the pump efficiency decreases with the pumping ratio. The pump efficiency is $62.66 \%$ for $R_{p}$ $=0.2$ and $54.6 \%$ for $R_{p}=0.5$ when the loss is negligible.

Figure 3 shows the calculated mean wavelength as a function of the total pump power for these two significant pumping ratios of 0.2 and 0.5 , respectively. The results show that for $R_{p}=0.5$, the pump power independent meanwavelength operation, i.e., $\partial \bar{\lambda} / \partial P_{\text {total }}=0$, does not exist for large pump power of this fiber source. However, for $R_{p}$ $=0.2$, the pump-power-independent mean-wavelength operation was obtained when the pump power was approximately $75 \mathrm{~mW}$.

On the basis of the above simulations, the mean wavelength and output power against the total pump power for a pumping ratio of 0.2 and EDF length of $19 \mathrm{~m}$ were measured, and the results are shown in Fig. 4. The experimental results are in good agreement with the simulation. The pump-power-independent mean-wavelength operation was observed when the pump power was $82 \mathrm{~mW}$. The inset in Fig. 4 shows the output L-band SFS spectrum for a pump power of $82 \mathrm{~mW}$. The total SFS output power is $34 \mathrm{~mW}$ with a linewidth of $42.6 \mathrm{~nm}$.

By replacing a pair of pump diodes by a single laser diode in the bidirectional pumped configuration, a L-band SFS with pump-power-independent mean-wavelength operation was achieved by optimization. From the L-band SFS spectrum inset in Fig. 4, it can be seen that the L-band SFS spectrum has two humps with the double-pass bidirec-

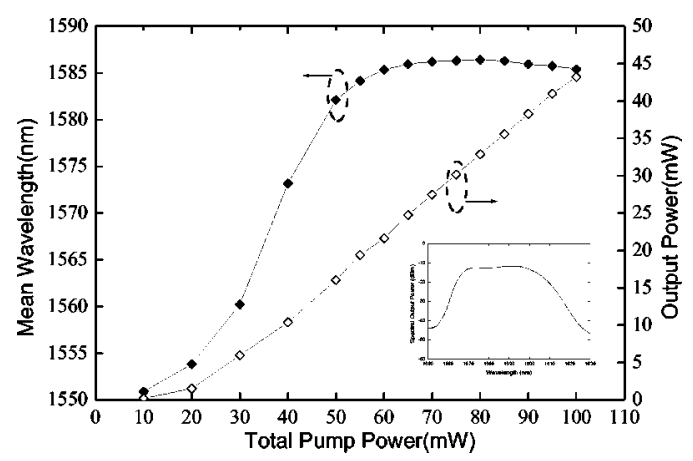

Fig. 4 Measured output power and mean wavelength versus pump power with $R_{p}=0.2$ and $L_{\mathrm{EDF}}=19 \mathrm{~m}$. Inset: SFS spectrum for a pump power of $82 \mathrm{~mW}$. tional pumped configuration; one is around $1570 \mathrm{~nm}$ and the other is around $1600 \mathrm{~nm}$. Synchronous changes of the backward and forward pump power with a proper pumping ratio ensure no fluctuation between the two humps as pump power increases in a certain pump power region, thus the mean wavelength variation versus the pump power is reduced to zero. Namely, the population distribution in the upper state remains unchanged with pump power variation (i.e., the backward pump and forward pump variation are synchronous) in a certain pump power region with a proper pumping ratio. However, the population distribution cannot remain unchanged with pump power variation of the backward pump or forward pump, respectively. The population distribution in the upper state determines the emission spectrum. Therefore, the unchanged distribution implies that a SFS with mean-wavelength stability can be obtained. As the spectral output power of the SFS is affected by the reflectivity of the fiber mirror, ${ }^{7}$ the optimization values of the EDF length and pumping ratio are dependent on the reflectivity of the fiber mirror.

\section{Conclusion}

In conclusion, we have characterized a practical design of the L-band SFS of a bidirectional pump configuration. By ensuring pump power variations of the forward and backward pump simultaneously with a power splitter, the $\partial \bar{\lambda} / \partial P_{\text {total }}$ instability, a limiting problem for high-precision fiber optic gyroscopes, can be reduced to zero for a chosen EDF length by optimizing the pumping ratio. A meanwavelength stability L-band SFS with a linewidth of 42.6 $\mathrm{nm}$, an output power of $34 \mathrm{~mW}$ was experimentally obtained. Advantages of the proposed L-band SFS are high mean-wavelength stability, broad linewidth, and high output power.

\section{Acknowledgments}

This work was supported by the Natural Science Foundation of Fujian Province under Grant No. A0440009 and Fujian Provincial Key Laboratory of Photonic Technology under Grant No. FP0408.

\section{References}

1. P. F. Wysocki, M. J. F. Digonnet, B. Y. Kim, and H. J. Shaw, "Characteristics of erbium-doped superfluorescent fiber sources for interferometric sensor applications," J. Lightwave Technol. 12(3), 550567 (1994).

2. C. D. Su and L. A. Wang, "Multiwavelength fiber sources based on double-pass superfluorescent fiber sources," J. Lightwave Technol. 18(5), 708-714 (2000).

3. L. A. Wang and C. D. Chen, "Stable and broadband Er-doped superfluorescent fiber sources using double-pass backward configuration," Electron. Lett. 32(19), 1815-1817 (1996).

4. B. Yang, H. Ming, Y. Guo, and J.-P. Xie, "Flattening spectrum of a broadband and wavelength stable erbium doped superfluorescent fiber source," Chin. Phys. Lett. 16(5), 356-357 (1999).

5. P. Z. Zatta and D. C. Hall, "Ultra-stability two-stage superfluorescent fiber source for fiber optics gyroscope," Electron. Lett. 38(9), 406408 (2002).

6. L. A. Wang and C. D. Chen, "Characteristics comparison of Er-doped double-pass superfluorescent fiber sources pumped near $980 \mathrm{~nm}$," IEEE Photonics Technol. Lett. 9(4), 446-448 (1997).

7. S.-C. Tsai, T.-C. Tsai, P.-C. Law, and Y.-K. Chen, "High pumpingefficiency L-band erbium-doped fiber ASE source using double-pass bidirectional-pumping configuration," IEEE Photonics Technol. Lett. 15(2), 197-199 (2003).

8. X. Wang and W. Huang, "High efficiency and stability L-band erbium-doped superfluorescent fiber source," Proc. SPIE 5280, 221224 (2003).

9. OASIX v3.0: Lucent Technologies erbium doped fiber devices simulation software. 